\title{
Control of the atom (nucleus) lifetime in the excited state by means of a low-frequency external field
}

\author{
I D Feranchuk ${ }^{1}$, L I Komarov ${ }^{1,3}$ and A Ulyanenkov ${ }^{2}$ \\ ${ }^{1}$ Belorussian State University, F. Skariny Av., 4, 220050 Minsk, Republic of Belarus \\ ${ }^{2}$ Bruker AXS, Östl. Rheinbrückenstr. 50, 76187 Karlsruhe, Germany
}

Received 15 April 2002

Published 16 September 2002

Online at stacks.iop.org/JPhysB/35/3957

\begin{abstract}
The radiative decay dynamics of an atomic (nuclear) excited state split into two neighbouring sublevels are considered for the case of interaction with a low-frequency electromagnetic field. The conditions of cancellation of the spontaneous emission in this system are analysed beyond the rotating wave approximation.
\end{abstract}

\section{Introduction}

The characteristics of the radiation decay of quantum systems are not fully defined by their intrinsic properties but also depend on the parameters of the environment, as shown by Purcell [1] for the first time. Realization of this idea has been of great interest in quantum optics during the last decades. The modification of the spontaneous decay of an atom can be achieved by several different mechanisms and has been considered by many authors.

One of these mechanisms is a transformation of the eigenfunctions of the vacuum electromagnetic field or its density of states, if the atom radiates in the resonator, waveguide or in the medium with proper dielectric properties (for example, some papers on this subject are cited in [2]). Another mechanism leading to the formation of the atomic excited levels with a small radiation width (RW) ('dark' states) is determined by the interference of the states arising due to translational movement of the atom in a laser trap. The main investigation of this case is presented in the papers by the Cohen-Tannoudji school (for example, see [3] and citations therein).

There is one more way to modify the evolution law of the excited quantum system, which is the main focus of interest in this paper. The atom interacts with the oscillating external field and the control of the decay process is conditioned by the population modulation of the excited state. One of the first attempts to study this phenomenon is given in [4], where we show that the process of decreasing the level width for the quantum system in the temporal periodical field is quantitatively analogous to the well known effect of the anomalous absorption of the x-rays (Borrmann effect [5]) or to the phenomenon of the suppression of the nuclear reactions [6] which occur in the spatial periodical field within the crystals. In the framework of 
the Weisskopf-Wigner approximation, the general condition for the deceleration of the decay rate has been found, which can be applied to both quantum optics and other radiative relaxation processes.

The effects related to the variation of the atomic lifetime in the modulating field have been widely studied (see citations in [7]) and recently the first experimental observation of spontaneous emission cancellation [8] has been reported. The theory of this phenomenon is discussed in $[9,10]$ and references therein. In this paper we emphasize the important aspects of the control of the spontaneous decay which can be essential for some applications. It has been supposed, in the majority of papers on this subject, that the atom (molecule) contains a natural set of the excited levels which are necessary for the resonant interaction with the modulating laser field. However, the possibility of controlling the decay rate of the quantum system should not be restricted by quantum optics only. In particular, the artificial narrowing of the emission line width in the Mössbauer nucleus is of great interest for the solution of the inverse population problem for a gamma laser based on long-lived isomers [11,12]. In this case, it is rather difficult to realize even the simplest three-level scheme [10] with natural nuclear levels because of the lack of coherent modulating radiation in the ultraviolet and x-ray range. However, the necessary sequence of levels can be artificially created, for example, by means of Zeemann splitting of the excited state in the Mössbauer nucleus. The energy interval between the split sublevels in this situation is essentially less than that for the main transition, and the modulating field of the radio- or superhigh-frequency range can be used [12].

Another point is the validity of the rotating wave approximation (RWA) for the qualitative description of the phenomenon. In most of the above-mentioned papers, this approximation is used to construct the 'dressed' excited states of the atom in a modulating field [10]. The exact solution, obtained in [13] for the interaction between a two-level system and a quantum field, demonstrates that the 'dressed' states can differ essentially from those which correspond to the RWA. Therefore, we carry out the quantitative analysis of the control conditions for the quantum system lifetime beyond the RWA, which is important for some possible applications of the phenomenon. This paper is organized as follows: in section 2 we obtain the equation for the RW of the three-level quantum system in the modulating field using the Weisskopf-Wigner approximation, however, beyond the RWA. In section 3 the upper bound for the RW of the excited state of the quantum system is calculated using the method developed in [13], and some applications of the general formula are considered.

\section{Formulation of the problem}

The atom (or nucleus) is modelled in this work as a three-level quantum system (figure 1) situated in the external low-frequency field (ELF). The radiative relaxation of the atom from two neighbouring excited sublevels $(\uparrow, \downarrow)$ to the ground state $(0)$ is defined by the interaction between the atom and the vacuum electromagnetic field (VEF) and forms the subject of our investigation. The states $(\uparrow, \downarrow)$ can exist in the natural set of the atom levels or can be created artificially, for example, by means of Zeeman splitting of the atomic excited state in the homogeneous magnetic field. The periodical ELF is supposed to be quite intensive and resonant with respect to the transition $(\uparrow \leftrightarrow \downarrow)$. The ELF frequency is supposed to be essentially less than the frequency of the transition to the ground state. Then the excited sublevels can be considered as a two-level system in the quantum ELF. As discussed in many of the papers mentioned in the introduction, the qualitatively new superposition of the atomic states is formed at some values of the field parameters when the atom can be 'frozen' in the excited state, i.e. its effective lifetime essentially exceeds the lifetime of the initial sublevels $(\uparrow, \downarrow)$. The physical basis of this phenomenon is due to the decrease of the effective dipole moment which defines the interaction between the atom and VEF (see below). 


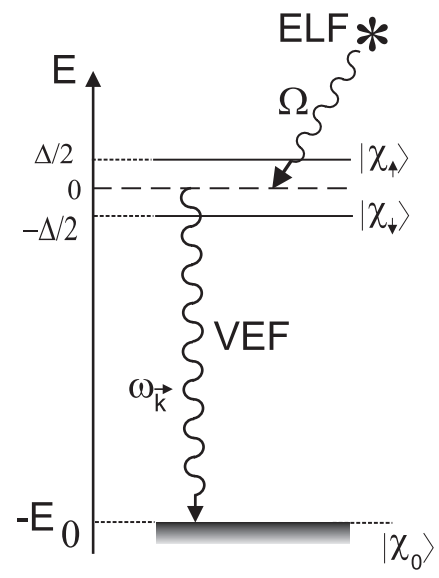

Figure 1. Scheme of the three-level system (see the notations in the text).

The RW within the framework of perturbation theory developed on the basis of the fine structure constant is defined by the interaction between the atom and VEF. The RW is performed [14] as quasi-stationary solutions (Weisskopf-Wigner approximation) of the Schrödinger equation with the following Hamiltonian of the model:

$$
\begin{aligned}
& \hat{H}|\psi\rangle=E|\psi\rangle ; \\
& \hat{H}=\hat{H}_{a}+\hat{H}_{r}+\hat{H}_{e}+\hat{V}_{r}+\hat{V}_{e} .
\end{aligned}
$$

For the three-level scheme (figure 1) the various parts of the total Hamiltonian $\hat{H}$ for the non-relativistic case are

$$
\begin{aligned}
& \hat{H}_{a}=-E_{0}\left|\chi_{0}\right\rangle\left\langle\chi_{0}\right|+\frac{1}{2} \Delta \sigma_{3} ; \\
& \hat{H}_{e}=\Omega a^{+} a ; \quad \hat{V}_{e}=f \sigma_{1}\left(a+a^{+}\right) ; \\
& \hat{H}_{r}=\sum_{k, s} \omega_{k} b_{k, s}^{+} b_{k, s} ; \\
& \hat{V}_{r}=-\frac{e}{m} \sum_{k, s}\left(\frac{2 \pi}{V \omega_{k}}\right)^{1 / 2} \mathrm{e}^{\mathrm{i} k r}\left(\boldsymbol{e}_{k, s} \hat{\boldsymbol{p}}\right)\left(b_{-k, s}^{+}+b_{k, s}\right) .
\end{aligned}
$$

Here we use the system of units with $\hbar=c=1$ and the frequency of the external field $\Omega$ defines the energy scale in the system. The value $-E_{0}\left(E_{0}>0\right)$ is the ground state energy of the atom and corresponds to the vector $\left|\chi_{0}\right\rangle ; \Delta$ is the energy-splitting of the excited state vectors $\left|\chi_{\uparrow, \downarrow}\right\rangle$ with energies $\pm \frac{1}{2} \Delta$, and Pauli matrices $\sigma_{i}$ operating in their basis; the coupling constant $f$ for the atom and ELF interaction is proportional to the dipole moment for transition between excited states; $a, a^{+}$are the annihilation and creation operators of the ELF quanta; $b_{k, s}^{+}, b_{k, s}$ are the creation and annihilation operators of the VEF photons with the wavevector $\boldsymbol{k}$, frequency $\omega_{k}$ and polarization $e_{k, s}$; the charge, mass and momentum operators for the atomic electron are denoted by $e, m, \hat{p}$, respectively; and $V$ is the normalized volume.

For further calculation we define a new basic set for the atomic excited states taking accurately into account the interaction of the atom with the quantum external field. In accordance with the terminology of quantum optics, these states correspond to the 'dressed' atom [10] and are the exact solutions of the following equation [13]:

$$
\left\{\frac{1}{2} \Delta \sigma_{3}+\Omega a^{+} a+f \sigma_{1}\left(a+a^{+}\right)-E_{n p}\right\}\left|\Psi_{n p}\right\rangle=0 .
$$


Thus, instead of two initial excited levels, the 'dressed' atom has a spectrum of states with quasi-energies $E_{n p}$ depending on the quantum number $n$ related to the quantity of photons in the field, and the quantum number $p= \pm 1$ depending on the combined parity operator [13], which commutes with the Hamiltonian of the two-level system in the quantum field

$$
\hat{P}\left|\Psi_{n p}\right\rangle \equiv \sigma_{3} \mathrm{e}^{\mathrm{i} \pi a^{+} a}\left|\Psi_{n p}\right\rangle=p\left|\Psi_{n p}\right\rangle .
$$

The RWA has been mainly used to obtain the solution of equation (4) and it results in quite simple analytical formulae for the quasi-energies and the states of the 'dressed' atom. However, according to our results [13], the RWA is actually suitable in the range of small values $f \sqrt{n} / \Omega$. Thus, the corrections to the quasi-energy states determined by the 'anti-rotating' terms in equation (4) can be comparable with the RW of the excited states. Therefore, we use the exact representation for the solutions of equations (4), (5) in the following form [13]:

$$
\left|\Psi_{n p}\right\rangle=\sum_{k=0}^{\infty} \sum_{q= \pm} C_{k q}^{n p}|k, f\rangle\left|\chi_{q}\right\rangle .
$$

Here the coefficients $C_{k q}^{n p}$ of the series are calculated for any quantum numbers and the entire range of the coupling constant $f$ by using the quickly convergent recurrent procedure described in [13]. The state vectors in the series are

$$
\begin{aligned}
& |n, f\rangle=\frac{\left(a^{+}+f / \Omega\right)^{n}}{\sqrt{n !}} \sum_{k=0}^{\infty} \frac{f^{k}}{k !}\left(a^{+}\right)^{k}\left|0_{a}\right\rangle \mathrm{e}^{-f^{2} / 2} ; \\
& a\left|0_{a}\right\rangle=0 ;\left|\chi_{ \pm}\right\rangle=\frac{1}{\sqrt{2}}\left(\left|\chi_{\uparrow}\right\rangle \pm\left|\chi_{\downarrow}\right\rangle\right) .
\end{aligned}
$$

Then the RW for the 'dressed' atom in the excited state with quantum numbers $(n, p)$ can be calculated by perturbation theory with respect to the interaction operator between the atom and VEF $\hat{V}_{r}$ using quantum electrodynamics [14]. The zeroth approximation for the solution of the Schrödinger equation (1) for the whole system is

$$
\left|\psi_{n p}^{(0)}\right\rangle=\left|\Psi_{n p}\right\rangle\left|0_{b}\right\rangle ; \quad b_{k s}\left|0_{b}\right\rangle=0 .
$$

The lifetime of the excited state is defined by the transition of the atom to the ground state with the emission of the photon $k s$ corresponding to the excitation of VEF. We can neglect the interaction between ELF and the atom in the ground state due to the inequality $\Omega \ll E_{0}$. This means that the ELF turns to one of its stationary states with a definite number of photons $m$ when the atom transfers to the ground state. In the first approximation on the radiative interaction $\hat{V}_{r}$, the wavefunction of the system is defined by the linear combination

$$
\begin{aligned}
& \left|\psi_{n p}^{(1)}\right\rangle=\left|\psi_{n p}^{(0)}\right\rangle+\sum_{m k s} B_{m k s}\left|\psi_{m k s}\right\rangle ; \\
& \left|\psi_{m k s}\right\rangle=\left|\chi_{0}, m\right\rangle b_{k s}^{+}\left|0_{b}\right\rangle ; \\
& \left|\chi_{0}, m\right\rangle=\left|\chi_{0}\right\rangle \frac{1}{\sqrt{m !}}\left(a^{+}\right)^{m}\left|0_{a}\right\rangle .
\end{aligned}
$$

Under the same conditions, the coefficients $B_{m k s}$ are calculated straightforwardly from equation (1):

$$
\begin{aligned}
& B_{m k s}=-\frac{1}{m \Omega+\omega_{k}-E_{0}-E_{n p}-\mathrm{i} 0}\left\langle\chi_{0}, m\left|d_{k s} \mathrm{e}^{-\mathrm{i} k r}\right| \Psi_{n p}\right\rangle \\
& d_{k s}=-\frac{e}{m}\left(\frac{2 \pi}{V \omega_{k}}\right)^{1 / 2}\left(e_{k, s} \hat{\boldsymbol{p}}\right),
\end{aligned}
$$


and the non-zeroth correction to the energy of the excited state arises in second-order perturbation theory:

$$
E_{n p}^{(2)}=\delta E_{n p}-\mathrm{i} \frac{\Gamma_{n p}}{2}=-\sum_{m k s} \frac{\left|\left\langle\chi_{0}, m_{a}\left|d_{k s} \mathrm{e}^{-\mathrm{i} k r}\right| \Psi_{n} p\right\rangle\right|^{2}}{m \Omega+\omega_{k}-E_{0}-E_{n p}-\mathrm{i} 0} .
$$

Then the formula for RW of the quasi-energy state of the 'dressed' atom is

$$
\Gamma_{n p}=2 \pi \sum_{m k s}\left|\left\langle\chi_{0}, m_{a}\left|d_{k s} \mathrm{e}^{-\mathrm{i} k r}\right| \Psi_{n} p\right\rangle\right|^{2} \delta\left(m \Omega+\omega_{k}-E_{0}-E_{n p}\right) .
$$

When we use definition (6) for the wavefunction, the matrix element of the operator of the interaction between the 'dressed' atom and VEF can be rewritten with the matrix elements for transitions of the real atom:

$$
\left\langle\chi_{0}\left|d_{k s} \mathrm{e}^{-\mathrm{i} k r}\right| \Psi_{n p}\right\rangle=\sum_{l q} C_{l q}^{n p}\left\langle\chi_{0}\left|d_{k s} \mathrm{e}^{-\mathrm{i} k r}\right| \chi_{q}\right\rangle\langle m \mid l, f\rangle,
$$

where the overlap integral $\langle m \mid l, f\rangle$ between the various representations of the ELF states can be found analytically.

To express the matrix elements for the interaction of the atom with VEF, the dipole approximation is used. The exponents in matrix elements can be omitted, and the vectors proportional to the dipole moments are defined for the transitions which take a part in the radiative relaxation:

$$
\boldsymbol{d}_{\uparrow, \downarrow}=\frac{e}{m}\left\langle\chi_{0}|\hat{\boldsymbol{p}}| \chi_{\uparrow, \downarrow}\right\rangle .
$$

After standard summation over the polarizations and integration over the impulses of the emitted photons in formula (12), we obtain the following result:

$$
\begin{aligned}
& \Gamma_{n p}=\frac{2}{3} \sum_{m \Omega<\left(E_{n p}+E_{0}\right)}\left(E_{n p}+E_{0}-m \Omega\right) \\
& \left|\sum_{l}\langle l, f \mid m\rangle\left[C_{l+}^{n p}\left(\boldsymbol{d}_{\uparrow}+\boldsymbol{d}_{\downarrow}\right)+C_{l-}^{n p}\left(\boldsymbol{d}_{\uparrow}-\boldsymbol{d}_{\downarrow}\right)\right]\right|^{2} .
\end{aligned}
$$

Formula (15) is obtained within the limits of usual approximations used for the calculation of the radiative lifetime in the nonrelativistic approximation. In general form, the following fact should be taken into account: despite the fact that the level width is presented by the sum of positive definite terms, it does not come to the simple sum of the widths of the excited levels of a free atom. The width of the level also holds the contribution defined by interference of matrix elements of both transitions. The expression structure for RW allows one to determine the conditions under which the atom lifetime increases. Here we use the following chain of inequalities to quantify the value $\Gamma_{n p}$ :

$$
\begin{gathered}
0 \leqslant \Gamma_{n p} \leqslant \frac{2}{3}\left(E_{n p}+E_{0}\right) \sum_{m<\left(E_{n p}-E_{0}\right)}\left|\sum_{l}\langle l, f \mid m\rangle\left[C_{l+}^{n p}\left(\boldsymbol{d}_{\uparrow}+\boldsymbol{d}_{\downarrow}\right)+C_{l-}^{n p}\left(\boldsymbol{d}_{\uparrow}-\boldsymbol{d}_{\downarrow}\right)\right]\right|^{2} \\
\leqslant \frac{2}{3}\left(E_{n p}+E_{0}\right) \sum_{m=0}^{\infty}\left|\sum_{l}\langle l, f \mid m\rangle\left[C_{l+}^{n p}\left(\boldsymbol{d}_{\uparrow}+\boldsymbol{d}_{\downarrow}\right)+C_{l-}^{n p}\left(\boldsymbol{d}_{\uparrow}-\boldsymbol{d}_{\downarrow}\right)\right]\right|^{2} .
\end{gathered}
$$

In the latter expression, we perform both summations over $m$ and one of the summations over $l$ using the fact that the set $|m\rangle$ is complete and the vectors $|l, f\rangle$ are orthonormal. As a result, 
the upper bound for the width of the considered level of the 'dressed' atom can be represented in the following form:

$$
\begin{aligned}
& 0 \leqslant \Gamma_{n p} \leqslant \Gamma_{n p}^{(0)} \equiv \frac{2}{3}\left(E_{n p}+E_{0}\right)\left[\left|\boldsymbol{d}_{\uparrow}\right|^{2} D_{\uparrow}^{n p}+\left|\boldsymbol{d}_{\downarrow}\right|^{2} D_{\downarrow}^{n p}+2 \operatorname{Re}\left(\boldsymbol{d}_{\uparrow} \boldsymbol{d}_{\downarrow}^{*}\right) D_{ \pm}^{n p} ;\right. \\
& \Gamma_{\uparrow, \downarrow}=\frac{2}{3}\left(E_{0} \pm \Delta / 2\right)\left|\boldsymbol{d}_{\uparrow, \downarrow}\right|^{2} ; \\
& D_{\uparrow, \downarrow}^{n p}=\sum_{l}\left|C_{l+}^{n p} \pm C_{l-}^{n p}\right|^{2} ; \quad D_{ \pm}^{n p}=\sum_{l}\left[\left|C_{l+}^{n p}\right|^{2}-\left|C_{l-}^{n p}\right|^{2} .\right.
\end{aligned}
$$

Evidently the introduced definitions of $\Gamma_{\uparrow, \downarrow}$ coincide with the width of the excited levels of the free atom. The other quantities depend on the renormalized dipole moment of the 'dressed' atom due to the interference in the superposition state caused by the interaction of the atom with the external field.

\section{Calculation of the upper bound for radiation width}

The coefficients $C_{l q}^{n p}$ derived in the previous section along with equation (17) define the conditions under which the effective control of the atom's lifetime in the excited state is possible using ELF. For every value of quantum numbers $(n, p)$, these coefficients can be obtained numerically [13], but the analytical analysis of the control conditions is also of great interest. The analytical approximation for solutions of equation (4) was obtained [13] along with the exact numerical solution on the basis of the operator method (OM). This approximation remains valid both within the limit of low coupling $f / \Omega \ll 1$ RWA and for $f \sqrt{n} / \Omega \gg 1$ (classical intensive external field) and also in the intermediate parameter range. Such a uniformly suitable approximation for quasi-energies has the following form [13]:

$$
\begin{array}{ll}
E_{n p} \simeq\left(n+\frac{1}{2} q\right) \Omega-\frac{f^{2}}{\Omega}+\frac{1}{4} p \Delta\left(S_{n n}+S_{n+q, n+q}\right)-\frac{1}{2} q M ; & q=p(-1)^{n} ; \\
M=\left\{\left[\Omega-\frac{1}{2} \Delta(-1)^{n}\left(S_{n n}-S_{n+q, n+q}\right)\right]^{2}+\Delta^{2} S_{n, n+q}^{2}\right\}^{1 / 2} ; & \\
S_{k m}=(-1)^{m} \exp \left(-2 \frac{f^{2}}{\Omega^{2}}\right)\left(\frac{2 f}{\Omega}\right)^{k-m} \sqrt{\frac{m !}{k !}} L_{m}^{k-m}\left(\frac{4 f^{2}}{\Omega^{2}}\right) ; & m \leqslant k ; S_{k m}=S_{m k},
\end{array}
$$

where $L_{m}^{k}(x)$ are the Laguerre polynomials. The symmetric matrix $S_{m k}$ arises as a result of the exact integral of motion (5) in calculation of the quasi-energies of the 'dressed' atom. In the zeroth-order approximation of OM, the coefficients of the wavefunction expansion (6) are determined by the following relationships:

$$
\begin{aligned}
& C_{l+}^{n p}=A \delta_{l n}+B \delta_{l, n+q} ; \\
& C_{l-}^{n p}=p\left[\left(A S_{n n}+B S_{n, n+q}\right) \delta_{l n}+\left(A S_{n+q, n}+B S_{n+q, n+q}\right) \delta_{l, n+q}\right] ; \quad A=\gamma B ; \\
& \gamma=-\frac{p \Delta S_{n, n+q}}{2 \Omega-2 f^{2} / \Omega+p \Delta S_{n n}-E_{n p}}=-\frac{p \Delta S_{n, n+q}}{\frac{p \Delta}{2}\left(S_{n n}-S_{n+q, n+q}\right)-q \Omega+q M},
\end{aligned}
$$

where $\delta_{k l}$ is the Kronecker symbol. Finally, we obtain the expression for the upper bound of the 'quasi-energy' level width:

$$
\begin{aligned}
& \Gamma_{n p}^{(0)}=\frac{2}{3}\left(E_{n p}+E_{0}\right)|B|^{2}\left[\left|\boldsymbol{d}_{\uparrow}\right|^{2} D_{\uparrow}^{n p}+\left|\boldsymbol{d}_{\downarrow}\right|^{2} D_{\downarrow}^{n p}+2 \operatorname{Re}\left(\boldsymbol{d}_{\uparrow} \boldsymbol{d}_{\downarrow}^{*}\right) D_{ \pm}^{n p} ;\right. \\
& D_{\uparrow, \downarrow}^{n p}=\left[\gamma \pm p\left(\gamma S_{n n}+S_{n, n+q}\right)\right]^{2}+\left[1 \pm p\left(\gamma S_{n+q, n}+S_{n+q, n+q}\right)\right]^{2} ; \\
& D_{ \pm}^{n p}=\gamma^{2}+1-\left(\gamma S_{n n}+S_{n, n+q}\right)^{2}-\left(\gamma S_{n+q, n}+S_{n+q, n+q}\right)^{2} .
\end{aligned}
$$

Despite the relatively cumbersome form, this expression allows one to analytically obtain the conditions for the parameters of the low-frequency field when $\Gamma_{n p}^{(0)}=0$. Because of 
inequalities (16), the width estimation becomes exact in this case. This means that the given quasi-energy state is long-lived even for short-lived excited sublevels of the 'dressed' atom, at least within the dipole approximation. In what follows, we present the mentioned stationarity conditions in an explicit form for two limiting cases: (a) weak coupling $f / \Omega \ll 1$, but $n \gg 1$; $f \sqrt{n} / \Omega \leqslant 1$ and (b) strong coupling $f \sqrt{n} / \Omega \gg 1$.

(a) In the case of weak coupling one can find [13]

$$
\begin{aligned}
& S_{n n} \simeq(-1)^{n} ; \quad S_{n+q, n+q} \simeq(-1)^{n+q}=-(-1)^{n} ; \\
& S_{n, n+q}=S_{n+q, n} \simeq(-1)^{n+(1+q) / 2} T ; \quad T=\frac{2 f}{\Omega} \sqrt{n+\frac{1}{2}(1+q)} ;
\end{aligned}
$$$$
(-1)^{(1+q) / 2} T \Delta
$$

$$
\gamma \simeq-\frac{(-1)+\sqrt{(\Delta-\Omega)^{2}+\Delta^{2} T^{2}}}{(\Delta-\Omega)}
$$

$D_{\uparrow, \downarrow}^{n p} \simeq\left(\gamma^{2}+1\right)\left(2+T^{2}\right) \pm 2 q\left(\gamma^{2}-1\right) \pm 4 \gamma q T(-1)^{(1+q) / 2} ;$

$$
D_{ \pm}^{n p} \simeq-\left(\gamma^{2}+1\right) T^{2}
$$

Depending on ratio dipole moments, all the quasi-energy states are divided into two groups, so the width $\Gamma_{n p}^{(0)}$ vanishes in one of them only. For $d_{\uparrow}>d_{\downarrow}$ this group is composed of the states with $q=-1$ and for $d_{\uparrow}<d_{\downarrow}$, the group is conditioned by $q=1$. Since the parameter $\Gamma_{n p}^{(0)}$ is positively defined, its tendency to zero for the stationary state results in the following relationships (for $q=-1$ ):

$$
\boldsymbol{d}_{\uparrow}=\gamma^{2} \boldsymbol{d}_{\downarrow} ; \quad T=-\frac{2 \gamma}{1-\xi} ; \quad \xi \equiv \frac{d_{\downarrow}}{d_{\uparrow}}<1,
$$

which lead to the following conditions for the external field parameters:

$$
\frac{f^{2} n}{\Omega^{2}}=\frac{\xi}{(1-\xi)^{2}} ; \quad \Omega=2 \Delta .
$$

The conditions obtained depend on the quantum number of the quasi-energy state. Therefore, the 'freezing' efficiency of the decay of the atomic excited state is determined by the quasienergy-level weight in expansion of the system state vector over the wavefunctions (6). The most important case is when the external field can be considered as a semi-classical one with the average number of photons $n_{0} \gg 1$, which is proportional to the field amplitude [15]. In this case, the quasi-energy states $n \simeq n_{0}$ are mostly populated and the first formula in equation (23) can be considered as a condition for the low-frequency field amplitude.

As an example of a concrete system for the proposed lifetime control technique in the considered weak coupling limit, here we examine the $3 \mathrm{~d}$ and $3 \mathrm{~s}$ states of a hydrogen-like atom with nuclear charge $Z$. In the dipole approximation, these states decay [14] only to the level $2 \mathrm{p}$, with $\xi \simeq 0.31$. The distance between excited sublevels is determined by the fine splitting, therefore conditions (22) result in the following relations ( $\alpha$ is the fine structure constant):

$$
\Omega=\frac{2(Z \alpha)^{2}}{3^{4}} Z^{2} \alpha ; \quad f^{2} n_{0} \simeq 0.65 \Omega .
$$

(b) In the case of strong coupling the asymptotic formula [13] can be used:

$$
S_{k n} \simeq \frac{(-1)^{n} \Omega^{1 / 2}}{\sqrt{2 \pi f \sqrt{n}}} \cos \left[\frac{4 f \sqrt{n}}{\Omega}-\frac{\pi(k-n)}{2}-\frac{\pi}{4}\right] ; \quad n \gg 1 ;(k-n) \ll 1,
$$

and using equation (19), we obtain

$$
\gamma \sim \sqrt{\frac{2 \pi f \sqrt{n}}{\Omega}} \gg 1 ; \quad A \gg B,
$$


which means the equal population of excited sublevels of the atom in the intense field. In this case formula (20) for $\Gamma_{n p}^{(0)}$ becomes appreciably simpler:

$$
\Gamma_{n p}^{(0)}=\frac{2}{3}\left(E_{n p}+E_{0}\right)|A|^{2}\left[\left|\boldsymbol{d}_{\uparrow}+\boldsymbol{d}_{\downarrow}\right|^{2}\right] .
$$

Thus, in the case of strong coupling the condition of freezing decay is reduced to the following relation:

$$
\boldsymbol{d}_{\uparrow} \simeq-\boldsymbol{d}_{\downarrow} .
$$

Such a condition can be fulfilled, for example, for sublevels of the $2 \mathrm{p}^{3 / 2}$ state of a hydrogen-like atom. These sublevels correspond to the projection of the total moment of electron $m \pm 1$, and consequently the distance $\Delta$ between these sublevels is specified by the hyperfine interaction. The analogous limit case can be realized for Zeeman sublevels of the excited state of some Mössbauer nuclei [12].

\section{Discussion}

The Borrmann effect during x-ray and gamma-ray diffraction in crystals [5] is a well known example of how the quantum state interference influences the inelastic relaxation processes. The essential suppression of the radiation absorption in the medium in this case is due to its spatial periodicity. Similar effects can be created by time periodicity in a quantum system and system relaxation parameters can be controlled by means of external conditions.

Despite the fact that all the calculations have been carried out for a simple three-level model, the physical principles of analysis can be generalized for a similar effect in more complex systems and for other mechanisms of relaxation. The phenomenon of interference of states, modulated by means of a low-frequency field, can influence not only the processes of radiative decay of excited states but other decay processes in quantum systems as well. The important practical application of the reported effect is a possible solution to the socalled gamma laser dilemma [11] in the production of coherent radiation by Mössbauer nuclei transitions. The artificial decay 'freezing' of excited states is a necessary condition for creation of inverted population in gamma lasers based on long-lived isomers and the proposed method can essentially extend the ability to build a gamma laser [11].

\section{Acknowledgments}

The authors are grateful to the Belarussian Foundation for Fundamental Research (Grant Nos F-234/2000, F-295/2000) and Bruker AXS GmbH for the support.

\section{References}

[1] Purcell E M 1946 Phys. Rev. 69681

[2] Moore G T 1970 J. Math. Phys. 112679 Agarval G S 1975 Phys. Rev. A 11230 Kleppner D 1981 Phys. Rev. Lett. 47233 Yablonovich E 1987 Phys. Rev. Lett. 582059 Quang T et al 1997 Phys. Rev. Lett. 795238 Agarval G S and Gupta S D 1998 Phys. Rev. A 57667

[3] Dalibard J, Reynaud S and Cohen-Tannoudji C 1984 J. Phys. B: At. Mol. Phys. 174577 Wallis H, Dalibard J and Cohen-Tannoudji C 1992 Appl. Phys. B 54407

[4] Feranchuk I D 1981 Phys. Lett. A 83126 Feranchuk I D 1980 Zh. Tech. Fiz. Lett. 61350

[5] Borrmann G 1941 Z. Phys. 42157 
[6] Afanas'ev A M and Kagan Yu 1965 Zh. Eksp. Teor. Fiz. 48327

[7] Radmore P M and Knight P L 1982 J. Phys. B: At. Mol. Phys. 15561

Lewenstein M, Zakrzewski J and Mossberg T 1988 Phys. Rev. A 38808

Agarwal G S 1991 Phys. Rev. Lett. 67980

Harshawardhan W and Agarwal G S 1997 Phys. Rev. A 552165

[8] Xia H R, Ye C Y and Zhu S Y 1996 Phys. Rev. Lett. 771032

[9] Zhu S Y and Scully M O 1996 Phys. Rev. Lett. 76388

Agarwal G S 1997 Phys. Rev. A 552457

[10] Facchi P and Pascazio S 2000 Phys. Rev. A 62023804

[11] Baldwinn G C, Salem J C and Goldanskii V I 1981 Rev. Mod. Phys. 53687

Vusotzkii V I and Kuzmin R N 1989 Gamma Lasers (Moscow: Moscow University)

[12] Feranchuk I D 1985 Dissertation Belarussian University

[13] Feranchuk I D, Komarov L I and Ulyanenkov A P 1996 J. Phys. A: Math. Gen. 294035

[14] Heitler W 1936 The Quantum Theory of Radiation (Oxford: Clarendon)

[15] Akhiezer A I and Beresteckii V B 1969 Quantum Electrodynamics (Moscow: Nauka) 